Pathologe 2010 · [Suppl 2] 31:147-152 DOI 10.1007/s00292-010-1347-5

Online publiziert: 11 . September 2010

(c) Springer-Verlag 2010

S. Wagner ${ }^{1} \cdot$ J. Calzada-Wack ${ }^{2} \cdot$ M. Rosemann ${ }^{3} \cdot$ L. Becker $^{1} \cdot$ M. Tost ${ }^{2}$.

P. Silva-Buttkus ${ }^{2} \cdot$ T. Klein-Rodewald $^{2} \cdot$ H. Fuchs ${ }^{1} \cdot$ F. Neff ${ }^{2} \cdot$ M. Hrabé de Angelis ${ }^{1}$.

I. Esposito ${ }^{2,4}$

${ }^{1}$ Institut für Experimentelle Genetik (IEG), München

${ }^{2}$ Institut für Pathologie, Helmholtz Zentrum München, München/Neuherberg

${ }^{3}$ Institut für Strahlenbiologie (ISB), Helmholtz Zentrum München

${ }^{4}$ Institut für Allgemeine Pathologie und Pathologische Anatomie, Technische Universität München

\title{
Charakterisierung von ENU-Mausmutanten
}

\section{Tiermodelle für menschliche Erkrankungen mittels morphologischer und molekularer Methoden}

Für die Erforschung der ursächlichen Pathomechanismen menschlicher Krankheiten stellen Mausmodelle das Mittel der Wahl dar. Sie können durch Genmanipulation (Z. B. „Knock-out“/"Gene trap“) oder mittels Bestrahlung/chemischer Mutagenese erzeugt werden. Ersteres führt durch das Ausschalten eines bestimmten Gens zu einem bestimmten, evtl. unbekannten Phänotyp („backward genetics“), während man bei Letzteren über den Phänotyp auf die kausale Mutation schließt („forward genetics“). Viele Studien zeigen, dass $N$-ethyl- $N$-nitrosourea (ENU) für die chemische Mutagenese das Mittel der Wahl darstellt [14].

\section{ENU-Mauslinien als Modelle für menschliche Krankheiten}

ENU führt direkt und ohne vorherige Metabolisierung durch Übertragung seiner Ethylgruppe zu einer Alkylierung in allen 4 DNA-Basen. Hierdurch entsteht ein Basenaustausch und somit eine Punktmutation an einer beliebigen Stelle des Genoms, sofern keine DNA-Reparaturmechanismen stattfinden. Vorrangig wirkt ENU auf die DNA von prämeiotischen Stammzellspermatogonien (• Abb. 1), aber auch auf eine Vielzahl anderer somatischer Stammzellen. Außer der muta- genen verfügt ENU auch über eine ausgeprägte karzinogene Wirkung. Die so hergestellten Mausmodelle stehen seit mehreren Jahren Wissenschaftlern für Forschungszwecke zur Verfügung. Am Helmholtz Zentrum München wurde das Münchner ENU-Mausmutagenese-Projekt im Jahr 2000 gestartet.

\section{Etablierung von ENU-Mutanten}

Im Münchner ENU-MausmutageneseProjekt werden Mäusemännchen des Inzuchtstammes $\mathrm{C}_{3} \mathrm{HeB} / \mathrm{FeJ}\left(\mathrm{C}_{3} \mathrm{H}\right)$ mit einer Dosis von $90 \mathrm{mg} / \mathrm{kg}$ Körpergewicht
(KG) i.p. ENU in 3 wöchentlich auf einander folgenden Injektionen behandelt. Ziel ist die Etablierung neuer dominanter Mausmodelle für menschliche Erbkrankheiten mit dem Schwerpunkt metabolische Erkrankungen. Der $\mathrm{C}_{3} \mathrm{H}$-Inzuchtstamm toleriert ENU-Dosierungen von bis zu einmalig $200 \mathrm{mg} / \mathrm{kgKG}$ i.p; die behandelten Männchen bleiben zu einem hohen Prozentsatz fertil [9]. In unserem Projekt konnten wir mit der von uns verwendeten Dosierung eine Sterilitätsra-

S. Wagner und J. Calzada-Wack sind gleichberechtigte Erstautoren.
Tab. 1 Methode für detaillierte Phänotypisierung der Mausmodelle im Institut für Pathologie

Komplette morphologische Untersuchung

Fixation der Organe in $4 \%$ gepuffertem Formalin, Einbettung in Paraffin für die histologische Untersuchung mit Hämatoxylin-EosinFärbung
Analysierte Organe

Muskel- und Skelettsystem Lymphknoten, Milz, Thymus Gehirn, Zerebellum Herz, Arterien Schilddrüse, Nebenschilddrüse, Nebenniere Trachea, Lunge

Haut, Zähne

Niere, Harnblase Ösophagus, Magen, Dünndarm, Dickdarm, Pankreas, Leber Hoden, Nebenhoden Ovarien, Uterus, Vagina
Zusätzliche Methoden

- Röntgenanalyse („Digital X-ray") - Spezielle histochemische Färbungen

- (Masson-Trichrom, „Periodic acidSchiff" (PAS), Chloracetatesterase (CAE), Movat) usw. - Immunhistochemie - Morphometrie und quantitative Bildanalyse

- Elektronenmikroskopie [Rasterelektronenmikroskopie (SEM) - Transmissionselektronenmikroskopie (TEM)] 


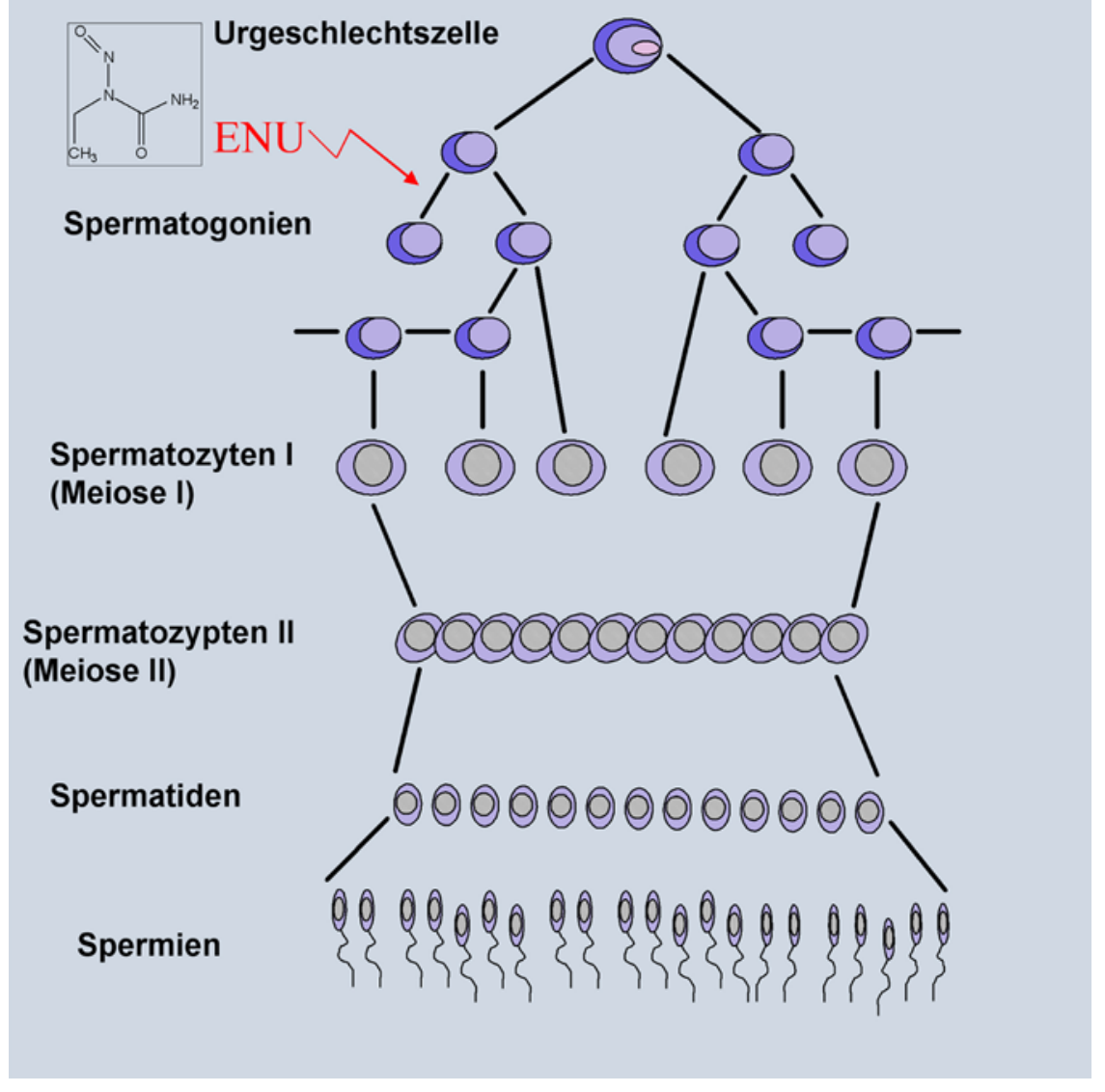

Abb. $1 \Delta$ Schematische Darstellung der Spermatogenese und Darstellung der Wirkung von ENU auf prämeiotische Stammzellspermatogonien

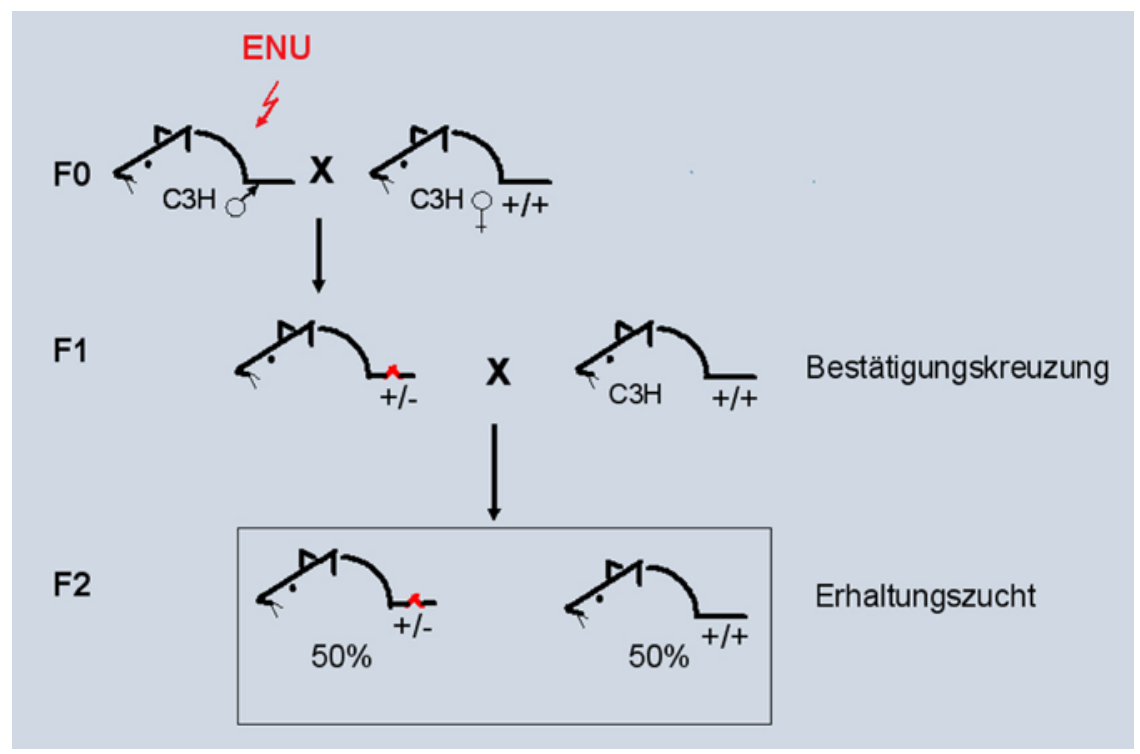

Abb. $2 \Delta$ Zuchtschema zur Bestätigung einer Mutation im dominanten Erbgang

te von etwa $52 \%$ beobachten. Nach statistischer Kalkulation ist zu erwarten, dass nach einer Injektion von $90 \mathrm{mg}$ ENU/ kgKG jedes F1-Tier etwa 20 Mutationen trägt [3].

Infolge der ENU-Behandlung ist eine Sterilitätsdauer von mehreren Wochen bis auch Monaten zu erwarten. Die behandelten Männchen werden 2 Wochen nach der Injektion verpaart, es werden aber nur Nachkommen phänotypisiert (F1-Generation), die mindestens 101 Tage nach der letzten ENU-Injektion geworfen wurden. Der Zyklus der Spermatogenese dauert bei der Maus 35 Tage [1] somit werden 2 vollständige Zyklen der Spermatogenese abgewartet, und es ist gewährleistet, dass diese Nachkommen nur von im Erbgut veränderten Spermien erzeugt wurden.

Wöchentlich werden Tiere der F1-Generation in verschiedenen Altersgruppen in einem standardisierten Arbeitsablauf nach blutbasierten und nichtblutbasierten Merkmalsveränderungen phänotypisiert. Die Blutentnahme erfolgt nach den Richtlinien des Tierschutzgesetztes und unter Genehmigung der Regierung von Oberbayern unter Ätheranästhesie bei 12 Wochen alten Mäusen durch Punktion des retroorbitalen Venenkomplexes. Im Plasma werden das Spektrum der gesamten klinischen Chemie (Olympus AU400, Olympus Hamburg), der Hämatologie, Immunologie und einige Parameter der Endokrinologie gemessen.

F1-Tiere, die im Vergleich zu WildtypMäusen veränderte Parameter mit mindestens 3 Standardabweichungen zeigen, werden nach 2 Wochen ein zweites Mal getestet. Bei Bestätigung des Phänotyps werden die auffälligen Tiere als so genannte „Founder-Tiere“ mit Wildtyp-Mäusen verpaart. Entsprechend des dominanten Erbgangs sind etwa 50\% Mutanten in diesen Bestätigungskreuzungen zu erwarten. Es werden 20 dieser Nachkommen getestet, und im Falle einer bestätigten Penetranz des Phänotyps wird eine Erhaltungszucht der neuen mutanten Linie etabliert (• Abb. 2).

Die Zucht über mindestens 10 Generationen gewährleistet, dass eine Selektion nur nach dem Phänotyp von Interesse geschieht und andere Mutationen herausgekreuzt werden. Die bestätigten Mauslinien erhalten bis zur Sequenzierung der genomischen Lokalisation der Mutation interne Namen. Ausgewählte Modelle werden im Institut für Pathologie am Helmholtz Zentrum München mittels verschiedener Techniken morphologisch und histologisch untersucht.

\section{Kartierung der Mutation}

Um eine Mutation genotypisch zu isolieren, erfolgt zunächst eine Rückzucht/ Auszucht auf einen anderen Inzuchtstamm. Wir verwenden in unserem Projekt $\mathrm{C}_{57} \mathrm{BL} / 6 \mathrm{~J}-\left(\mathrm{C}_{57}\right)$ Mäuse. $\mathrm{C}_{3} \mathrm{H}-\mathrm{Mu}-$ 
tanten werden mit Wildtyp-Tieren des C57-Stammes verpaart und die positiven Nachkommen nochmals auf C57 ausgekreuzt. Von den aus diesen zweiten Verpaarungen stammenden Nachkommen werden Schwanzspitzenbiopsien zur DNA-Extraktion genommen (• Abb. 3).

Nach der DNA-Extraktion erfolgt eine Grobkartierung der Lokalisation der Mutation mit einem Panel 158 gleichmäßig über das Genom verteilter SNPMarker mittels MALDI-TOF-Technologie (Sequenom, San Diego, CA, USA). Es folgt die Kandidatengensuche in der lokalisierten chromosomalen Region und anschließend deren Sequenzierung. Im Institut für Experimentelle Genetik am Helmholtz Zentrum laufen die Strategien der phänotypischen Charakterisierung von ENU-Mausmutanten und der genomischen Kartierung der verursachenden Mutationen parallel im Hochdurchsatzverfahren. Ist der Ort der Mutation am Ende genau bekannt, kann das betroffene Gen mit dem jeweiligen Phänotyp korreliert und auf dessen Funktion geschlossen werden. Viele dieser Mausmodelle wurden wissenschaftlichen Kollaborationen zur Verfügung gestellt und publiziert (z. B. $[4,7,16])$.

\section{Morphologische Untersuchungen ausgewählter Modelle}

Das Institut für Pathologie im Helmholtz Zentrum München beschäftigt eine Gruppe von Humanpathologen und Veterinärmedizinern, die langjährige Erfahrung in der morphologischen Phänotypisierung von Tiermodellen für menschliche Erkrankungen haben. Ziel ist es, das Verständnis zu erweitern, wie genetische Veränderungen die menschlichen Krankheiten beeinflussen. Darüber hinaus ist das Institut für Pathologie Teil der Deutschen Mausklinik („German Mouse Clinic“, GMC), die eine einzigartige Institution zur detaillierten Phänotypisierung mutanter Mauslinien in Deutschland darstellt (http://www.mouseclinic.de/).

Das Institut verfügt über eine gute Infrastruktur, die es ermöglicht, alle modernen diagnostischen Techniken, die in der Humanpathologie verwendet werden, auch für die Untersuchung von Nagetiermodellen zu benutzen. Die primäre Un-

Pathologe 2010 · [Suppl 2] 31:147-152 DOI 10.1007/s00292-010-1347-5

C) Springer-Verlag 2010

S. Wagner - J. Calzada-Wack · M. Rosemann - L. Becker · M. Tost · P. Silva-Buttkus ·

T. Klein-Rodewald $\cdot$ H. Fuchs $\cdot$ F. Neff $\cdot M$. Hrabé de Angelis $\cdot$ I. Esposito

Charakterisierung von ENU-Mausmutanten.

Tiermodelle für menschliche Erkrankungen mittels morphologischer und molekularer Methoden

Zusammenfassung

Nach der Entschlüsselung des Humangenoms besteht die Herausforderung, gezielte Kenntnisse über die genauen Genfunktionen und das Zusammenspiel dieser Gene und Signalwege bei der Entstehung von Krankheiten zu erlangen. Mausmodelle stellen hierfür das Mittel der Wahl dar. Die chemischen Mutagenese mittels $\mathrm{N}$-ethyl- $\mathrm{N}$-nitrosourea (ENU) ermöglicht durch die Erzeugung zufälliger Punktmutationen eine differenzierte Analyse der Auswirkungen eines einzigen Basenaustausches auf den gesamten Organismus. Das Münchner ENU-Mausmutagenese-Projekt hat sich der weltweiten Initiative angeschlossen, Einblicke in die Bedeutung einzelner Genabschnitte zu erhalten. Im Rahmen eines genomweiten syste-

matischen Hochdurchsatz-Screenings wurden Mausmodelle für eine Vielzahl menschlicher Erbkrankheiten entwickelt. Diese Arbeit verdeutlicht, wie die Implementierung des ENU-Mausmutagenese-Projekts und der Genidentifikation im parallelen Hochdurchsatz-Screening, unter der Möglichkeit der engen örtlichen Zusammenarbeit mit erfahrenen Phänotypisierungsgruppen am Helmholtz Zentrum München, zu wesentlichen Fortschritten in der funktionellen Analyse des Säugetiergenoms führen kann.

\section{Schlüsselwörter}

$\mathrm{N}$-ethyl-N-nitrosourea (ENU) · ENU-

Mutagenese - Mausmodelle $\cdot$ Morphologische Phänotypisierung

\section{Characterization of ENU-mutant mice. Animal models for human diseases using morphological and molecular methods}

Abstract

Following sequencing of the human genome there are new challenges to decipher the knowledge concerning gene function and the role of gene interactions and pathways leading to disease. Mouse models have proven to be an ideal tool for this purpose. Point mutations induced by chemical mutagenesis by $\mathrm{N}$-ethyl- $\mathrm{N}$-nitrosourea (ENU) offer possibilities for the analysis of the phenotypic outcome of a single base pair exchange on the entire organism. The Munich ENU mouse mutagenesis project is part of the worldwide efforts to obtain mutations for each gene. The generation of new alleles or allelic series offers relevant insights into the relevance of single gene sections. Various mouse models for human diseases have been generated by a systematic large-scale genome-wide phenotyping screen in the last decade. This work illustrates how the implementation of the ENU mouse mutagenesis project with gene identification and parallel high-throughput screening is taking advantage of local cooperation with experienced phenotyping groups at the Helmholtz Zentrum München, leading to major advances in the functional analysis of the mammalian genome.

\section{Keywords}

$N$-ethyl- $N$-nitrosourea (ENU) · ENU mutagenesis · Mouse models · Morphologic phenotyping 


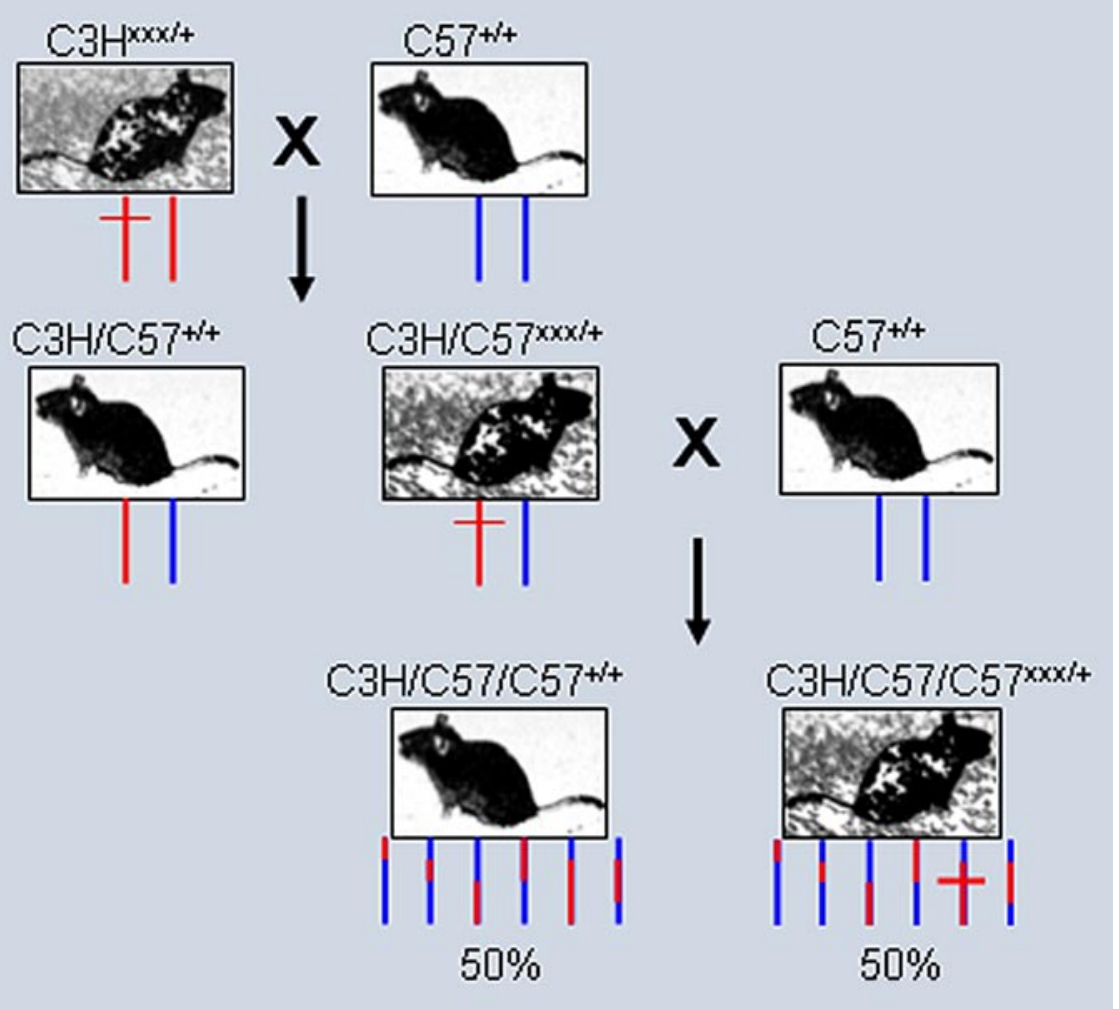

Abb. $3 \Delta$ Auszucht einer Mutation auf einen zweiten Inzuchtstamm. In der zweiten Zuchtphase findet das genomische "Crossing over" statt

tersuchung der ENU-Mauslinien besteht aus einer kompletten morphologischen Untersuchung. Dazu werden 10 männliche $(\mathrm{m})$ und 10 weibliche $(\mathrm{w})$ mutierte Mäuse mit ihren normalen Wurfgeschwistern (10 $\mathrm{m}$ und $10 \mathrm{w}$ ) verglichen. - Tab. 1 gibt einen Überblick über die von uns eingesetzten Methoden und routinemäßig analysierten Organe.

In den Fällen, in denen die morphologischen Daten allein nicht ausreichen, um zu einer schlüssigen Diagnose zu gelangen, ist die Immunhistochemie (IHC) eine entscheidende technische Hilfe, um Proteinexpressionsprofile auf paraffineingebetteten oder gefrorenen Gewebeschnitten zu bestimmen. Die IHC einzeln oder als Doppelimmunfärbung wird mit einem Färbeautomat (Discovery, Roche Deutschland $\mathrm{GmbH}$ ) durchgeführt. IHCProtokolle wurden mit verschiedenen Reagenzien und mehr als 61 spezifischen Antikörpern für Nagetiergewebe etabliert. Die positive Immunfärbung kann dann mit einer Bildanayse-Software kombiniert werden, um die Daten quantitativ zu analysieren. Auch ein Imaging-System für die „virtuelle Mikroskopie“ (Olympus dotslide, Münster, Deutschland), welches Dis- kussionen und weltweite Konsultationen ermöglicht, ist vorhanden. Ein weiteres von uns häufig benutztes Instrument zur Erkennung der Ultrastruktur einzelner Zellen ist das Transmissionselektronenmikroskop (TEM). Das breite Spektrum an Techniken, die im Institut für Pathologie zur Charakterisierung von Tiermodellen angewendet werden, ist exemplarisch in - Abb. 4 a, b, - Abb. 5 a-c und 6 dargestellt.

\section{Neurologische Untersuchungen ausgewählter Modelle}

Der neurologische Primär-Screen im Institut für Neurologie beinhaltet eine Reihe von Untersuchungen zur Feststellung grundlegender neurologischer Funktionen. Analog zur medizinischen Diagnostik wird anhand eines „SHIRPAProtokolls" (http://www.har.mrc.ac.uk/ services/phenotyping/neurology/shirpa. html) eine Serie von standardisierten Tests und Beobachtungen durchgeführt, um Auffälligkeiten zu detektieren, Reflexe, Gang, Körpertonus und Aktivitätsparameter zu überprüfen sowie einen Gesamteindruck über den Gesundheitsstatus zu ermöglichen. Zusätzlich wird über eine spezielle Haltevorrichtung für die Vorderextremitäten eine Kraftmessung durchgeführt. Die Bewegungskoordination wird mit einem Rotarod-Test überprüft. Da die Rotationsgeschwindigkeit allmählich zunimmt, müssen die Tiere ihre Bewegung immer neu und mit zunehmender Schwierigkeit anpassen. So kann die Fähigkeit zur Koordination über die Zeit, in der sie entsprechend mitlaufen können, quantifiziert werden [5]. Je nach Protokoll und Wiederholungsmessungen können so auch Ermüdung und/oder motorisches Lernen gemessen werden. Ausgehend von den Ergebnissen dieser Eingangstests können dann gezielt weitergehende Analysen wie z. B. eine automatische Ganganalyse oder elektrophysiologische Messungen (z. B. EMG, evozierte Potenziale, EEG) durchgeführt werden.

\section{Diskussion}

Im Laufe des Münchner ENU-Mutagenese-Projekts konnten erfolgreich viele Mausmodelle für menschliche erbliche Erkrankungen etabliert werden (• Tab. 2).

Zusätzlich bieten Studien an Mausmodellen die Möglichkeit, die Effekte von Punktmutationen auf molekulare Signalwege zu analysieren und Zusammenhänge von Geninteraktionen nachzuvollziehen. Einige der Modelle aus dem Münchner ENU-Mutagenese-Projekt wurden auch in der GMC und im Modul der Pathologie detailliert charakterisiert und phänotypisiert. Gerade hinsichtlich der Pathologie bieten Mausmodelle die unschätzbaren Möglichkeiten, die Auswirkungen von Mutationen auf den gesamten Organismus zu analysieren. Mit Hilfe dieser Strategie war es unlängst u. a. möglich, eine neue Mauslinie mit neurologischem Phänotyp (Kachexie, Ataxie, Tremor) zu etablieren, die als Modell für ein Krankheitsbild entsprechend einer hereditären sensorischen und motorischen Neuropathie (HSMN) dienen könnte. Die SNP-Analyse zeigte eine Lokalisation bei den Markern rs26982471 und rs27041242 aufChromosom 11 (53.99-98.55 Mb, Build 37.1).

Die hier vorgestellten Beispiele aus dem Münchner ENU-Mausmutagenese-Projekt verdeutlichen, wie die Zusam- 
Abb. $4>$ a Anti-Neurofilament- (NF-) Protein-Immunhistochemie am Gehirn (koronale Abschnitte). Der Antikörper gegen das humane Protein zeigt Kreuzreaktivität mit dem NF-äquivalenten Protein in der Maus und färbt die in der weißen Substanz gelegenen Axone. b Ki-67-Immunhistochemie eines Follikels im zyklischen Ovar zur Berechnung des Proliferationsindexes
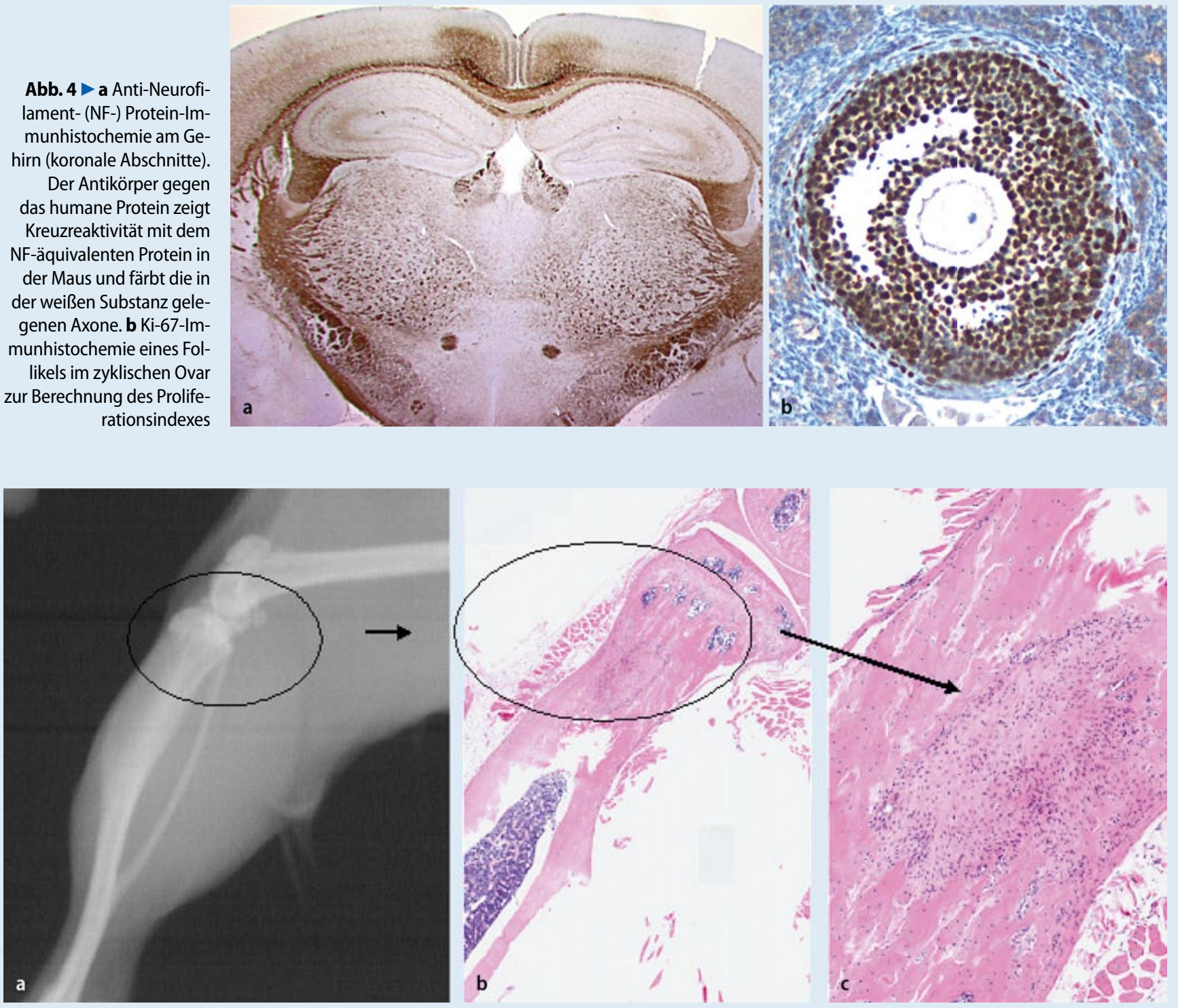

Abb. $5 \Delta$ Gegenüberstellung von Röntgtenbild (a) und histologischer Darstellung (b, c) der Knochenläsion einer Maus

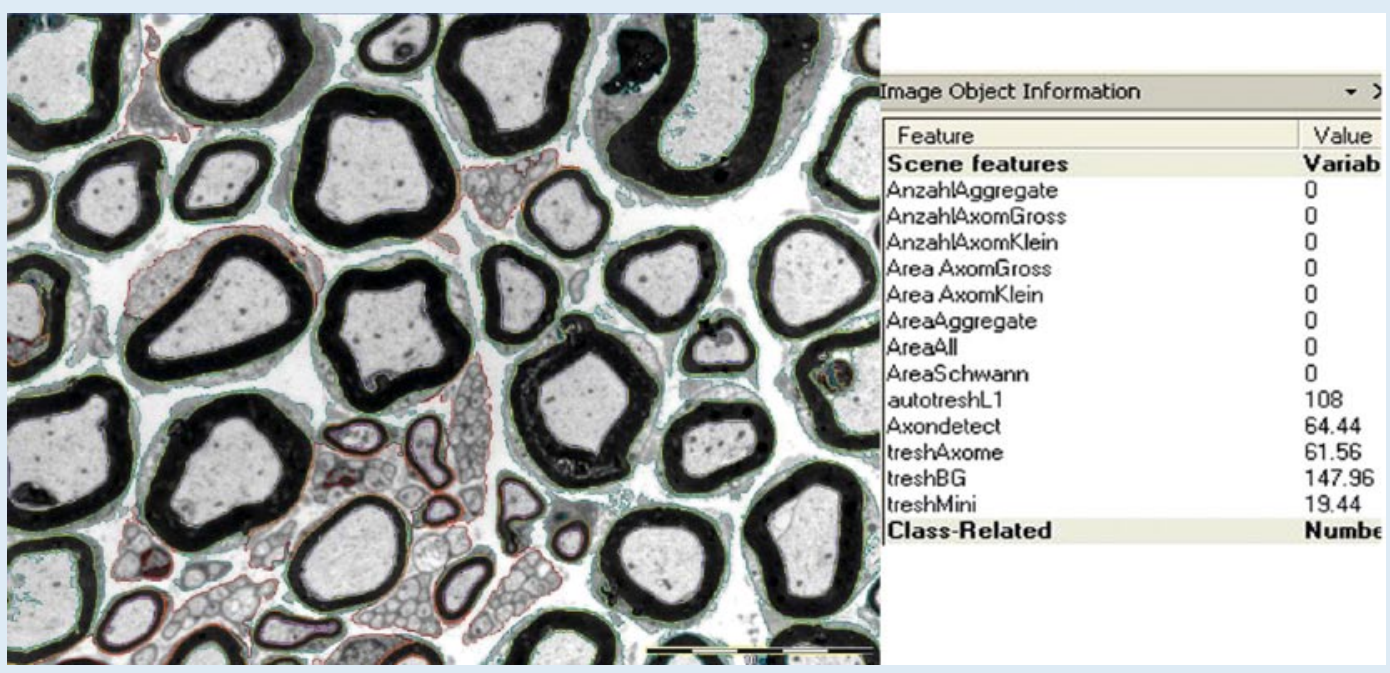




\begin{tabular}{|lll}
\hline Tab. 2 & Auswahl von ENU-Mausmodellen für menschliche erbliche Erkrankungen \\
\hline Gen & Modell für & Referenz \\
\hline Col1a1 & Osteogenesis imperfecta & {$[10]$} \\
\hline Crygd & Katarakt & {$[6]$} \\
\hline Enamelin & Amelogenesis imperfecta & {$[15]$} \\
Gjf1 & Mikrophthalmie & {$[13]$} \\
Ins2 & Diabetes & {$[8]$} \\
\hline Kit & Pigmentationsstörung mit metabolischem Effekt & {$[11]$} \\
Rsp19 & Diamond-Blackfan-Anämie & {$[12]$} \\
Rsp20 & Diamond-Blackfan-Anämie & {$[12]$} \\
Tcfap2a & Taubheit (Mittelohrdefekt) & {$[2]$} \\
\hline
\end{tabular}

menarbeit verschiedenster Gruppen des Helmholtz Zentrums zur Identifizierung medizinisch relevanter Phänotypen und der zugrunde liegenden Gene, analog zur Untersuchung von Patienten in der Klinik, beitragen kann.

\section{Korrespondenzadressen \\ Dr. J. Calzada-Wack \\ Institut für Pathologie, \\ Helmholtz Zentrum München \\ Ingolstädter Landstr. 1, \\ 85764 München/Neuherberg \\ calzada@helmholtz-muenchen.de}

\section{Prof. Dr. I. Esposito}

Institut für Pathologie,

Helmholtz Zentrum München

Ingolstädter Landstr. 1 ,

85764 München/Neuherberg

Danksagung. An die Mitarbeiter: Annette Feuchtinger, Luise Jennen und Elenore Samson für die exzellente technische Unterstützung.

Diese Arbeit wurde vom Nationalen Genomforschungsnetz (NGFN plus) unterstützt (Förderkennzeichen: 01GS0850).

Interessenkonflikt. Die korrespondierenden Autoren geben an, dass kein Interessenkonflikt besteht.

\section{Literatur}

1. Adler ID (1996) Comparison of the duration of spermatogenesis between male rodents and humans. Mutat Res 352:169-172

2. Ahituv N, Erven A, Fuchs H et al (2004) An ENU-induced mutation in AP-2alpha leads to middle ear and ocular defects in Doarad mice. Mamm Genome 15(6):424-432

3. Augustin M, Sedlmeier R, Peters T et al (2005) Efficient and fast targeted production of murine models based on ENU mutagenesis. Mamm Genome 16:405-413

4. Bosman EA, Quint E, Fuchs H et al (2009) Catweasel mice: a novel role for Six 1 in sensory patch development and a model for branchio-oto-renal syndrome. Dev Biol 328(2):285-296
5. Brooks SP, Dunnett SB (2009) Tests to assess motor phenotype in mice: a user's guide. Nat Rev Neurosci 10(7):519-529

6. Graw J, Neuhäuser-Klaus A, Löster J, Favor J (2002) A 6-bp deletion in the Crygc gene leading to a nuclear and radial cataract in the mouse. Invest Ophthalmol Vis Sci 43(1):236-240

7. Hafezparast M, Klocke R, Ruhrberg C et al (2003) Mutations in dynein link motor neuron degeneration to defects in retrograde transport. Science 300(5620):808-812

8. Herbach N, Rathkolb B, Kemter E et al (2007) Dominant-negative effects of a novel mutated Ins2 allele causes early-onset diabetes and severe betacell loss in Munich Ins2C95S mutant mice. Diabetes 56(5):1268-1276

9. Justice MJ, Carpenter DA, Favor J et al (2000) Effects of ENU dosage on mouse strains. Mamm Genome 11:484-488

10. Lisse TS, Thiele F, Fuchs $\mathrm{H}$ et al (2008) ER stress-mediated apoptosis in a new mouse model of osteogenesis imperfecta. PLoS Genet 4(2):e7

11. Magnol L, Chevallier MC, Nalesso V et al (2007) $\mathrm{KIT}$ is required for hepatic function during mouse post-natal development. BMC Dev Biol 7:81

12. McGowan KA, Li JZ, Park CY et al (2008) Ribosomal mutations cause $\mathrm{p} 53$-mediated dark skin and pleiotropic effects. Nat Genet 40(8):963-970

13. Puk O, Löster J, Dalke C et al (2008) Mutation in a novel connexin-like gene (Gjf1) in the mouse affects early lens development and causes a variable small-eye phenotype. Invest Ophthalmol Vis Sci 49(4):1525-1532

14. Russell WL, Kelly EM, Hunsicker PR et al (1979) Specific-locus test shows ethylnitrosourea to be the most potent mutagen in the mouse. Proc Natl Acad Sci U S A 76:5818-5819

15. Seedorf $\mathrm{H}$, Klaften $M$, Eke $F$ et al (2007) A mutation in the enamelin gene in a mouse model. J Dent Res 86(8):764-768

16. Vreugde S, Erven A, Kros CJ et al (2002) Beethoven, a mouse model for dominant, progressive hearing loss DFNA36. Nat Genet 30(3):257-258 